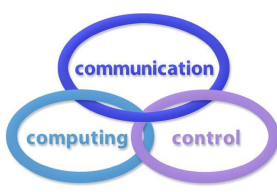

\title{
Crisp-linear-and Models in Fuzzy Multiple Objective Linear Fractional Programming
}

\author{
B. Stanojević, S. Dzitac, I. Dzitac
}

\author{
Bogdana Stanojević* \\ Mathematical Institute of the Serbian Academy of Sciences and Arts, Serbia \\ Kneza Mihaila 36, 11000 Belgrade, Serbia \\ *Corresponding author: bgdnpop@mi.sanu.ac.rs
}

\section{Simona Dzitac}

University of Oradea, Department of Energy Engineering,

Universitatii 1, 410087 Oradea, Romania

sdzitac@uoradea.ro

\section{Ioan Dzitac}

1. Aurel Vlaicu University of Arad

310330 Arad, Elena Dragoi, 2, Romania

ioan.dzitac@uav.ro

2. Agora University of Oradea

410526 Oradea, P-ta Tineretului 8, Romania,

idzitac@univagora.ro

3. University of Craiova, Department of Economic Informatics \& Statistics, 200585, Craiova, Str. A.I. Cuza, nr. 13, Romania

\begin{abstract}
The aim of this paper is to introduce two crisp linear models to solve fuzzy multiple objective linear fractional programming problems. In a novel manner we construct two piece-wise linear membership functions to describe the fuzzy goal linked to a linear fractional objective. They are related to the numerator and denominator of the fractional objective function; and we show that using the fuzzy-and operator to aggregate them a convenient description of the original fractional fuzzy goal is obtained. Further on, with the help of the fuzzy-and operator we aggregate all fuzzy goals and constraints, formulate a crisp linear model, and use it to provide a solution to the initial fuzzy multiple objective linear fractional programming problem. The second model embeds in distinct ways the positive and negative information, the desires and restrictions respectively; and aggregates in a bipolar manner the goals and constraints. The main advantage of using the new models lies in the fact that they are linear, and can generate distinct solutions to the multiple objective problem by varying the thresholds and tolerance limits imposed on the fuzzy goals.

Keywords: fuzzy linear fractional programming, fuzzy multiple objective programming, fuzzy decision, aggregation operator.
\end{abstract}




\section{Introduction}

Zimmermann [12] proposed a solution approach in fuzzy mathematical programming using BellmanZadeh extension principle [1]. He treated in a symmetrical way both the fuzzy goals and constraints, and solved linear programming problems with several objective functions. Later on, Zimmermann [13] discussed some aspects of duality and efficiency, emphasizing the importance of the applications of the fuzzy set theory to mathematical programming.

Recently, Dubey, Chandra and Mehra [4] introduced several crisp linear models in fuzzy multiple objective linear programming derived from using different fuzzy operators to aggregate the fuzzy goals and constraints of the initial problem. Separating the goals and constraints, and aggregating them in different ways a bipolar framework for computing a Pareto-optimal solution to multiple objective flexible linear programming is developed in [3]. Following their idea, Li, Leung, and $\mathrm{Wu}$ [7] solved multiple objective interval linear programming problems in the admissible-order vector space.

Linear fractional optimization problem was firstly introduced to solve a bi-objective linear programming problem. Maximizing a ratio can be seen a simultaneously maximization of the numerator and minimization of the denominator, its solution being one solution among many Pareto optimal solutions of the bi-objective problem. Later on, it was used to model directly real life problems. In the standard trade-off between accuracy and simplicity, fractional programming offers more accuracy, and in the same time succeeds to avoid the overloading of the model. However fractional objectives used in the classic membership functions of the fuzzy goals make them being non-linear.

In a wide variety of fuzzy approaches to solve multiple objective problems linear models are desired, thus efforts were made to linearize the membership functions. There are many papers in the literature devoted to modeling multiple objective fractional optimization problems as fuzzy decision problems, and solving them through fuzzy goal programming (see for instance the recent ones [2], [6], [10], [11]).

In this paper we propose a solution approach to multiple objective linear fractional programming (MO-LFP) problems based on fuzzy goal aggregation. We introduce a new pair of piece-wise linear membership functions that together describe a linear fractional fuzzy goal, and use them to model the multiple objective decision problem. The main advantage of the novel pair of membership functions is twofold: they are piece-wise linear, and their aggregation using min operator is in accordance to the aggregation used in solving classic fuzzy decision problems. The crisp-linear-and models we provide solve a MO-LFP problem with fuzzy goals and constraints firstly aggregated in a classic framework, and secondly in a bipolar framework.

In Section 2 we review some standard facts on fuzzy goals, their aggregation, and their usefulness in fuzzy mathematical programming. Section 3 presents our improvement in handling the fractional objective functions. It is worth pointing out that we approach the non-linearity of the fractional objectives by using two piece-wise linear membership functions whose aggregation is made in the same manner as it is usual for the fuzzy goals, thus making them to behave in the same way as a single membership function linked to a fuzzy goal. In Section 4 we introduce our crisp-linear-and models; and analyze their complexity and usefulness in solving MO-LFP problems. Section 5 provides a numerical illustration of the membership functions construction, and novel solution approach. Our conclusions and final remarks as well as our ideas for further research are inserted in Section 6.

\section{Preliminaries}

The general model for a multiple objective linear programming (MO-LP) maximization problem is given below.

$$
\begin{aligned}
\max & \left(c_{1}^{T} x, \ldots, c_{p}^{T} x\right) \\
\text { s.t. } & a_{i} x \leq b_{i}, i=1, \ldots, m, \\
& x \geq 0
\end{aligned}
$$


Similarly, the general model for a multiple objective linear fractional programming (MO-LFP) maximization problem is

$$
\begin{aligned}
\max & \left(\frac{c_{1}^{T} x+c_{1}^{0}}{d_{1}^{T} x+d_{1}^{0}}, \ldots, \frac{c_{p}^{T} x+c_{p}^{0}}{d_{p}^{T} x+d_{p}^{0}}\right) \\
\text { s.t. } & a_{i} x \leq b_{i}, i=1, \ldots, m, \\
& x \geq 0 .
\end{aligned}
$$

Symbol "max" is used in the sense of finding a non-dominated solution. Whenever some parameters are fuzzy quantities, inequalities are fuzzy or there are fuzzy goals formulated on the objective functions of a programming problem the problem is called fuzzy programming problem and usually " $\widetilde{\max }$ " is used instead of "max" to denote the optimization in a fuzzy sense. In Section 4 we will focus on solving fuzzy MO-LP and MO-LFP problems with fuzzy goals and fuzzy constraints.

The classic way to construct a fuzzy goal related to any kind of objective function $f$ that has to be maximized is to involve a threshold $(p)$ and a tolerated limit $(t<p)$ on the given threshold, and define the membership function $\mu_{f}^{\max }$ given in (1). If the function $f$ is to be minimized, the corresponding fuzzy goal is also defined in (1) through the membership function $\mu_{f}^{\min }$. In this case the tolerance limit $t$ on the threshold has to be greater than $p$.

$$
\mu_{f}^{\max }(f(x))=\left\{\begin{array}{rl}
0, & f(x)<t, \\
\frac{f(x)-t}{p-t}, & t \leq f(x) \leq p, \\
1, & f(x)>p,
\end{array} \quad \mu_{f}^{\min }(f(x))=\left\{\begin{aligned}
0, & f(x)>t, \\
\frac{f(x)-t}{p-t}, & p \leq f(x) \leq t \\
1, & f(x)<p .
\end{aligned}\right.\right.
$$

Due to the stated inequality between the threshold $p$ and tolerance limit $t, \mu_{f}^{\max }$ is an increasing function while $\mu_{f}^{\min }$ is a decreasing one. If function $f$ is linear the non-constant branches of its membership functions are linear. Similarly, if function $f$ is linear fractional, its membership functions defined as above have linear fractional branches. In Section 3 we will construct a pair of membership functions that are piece-wise linear and their aggregation properly describe a linear fractional fuzzy goal.

A fuzzy decision making problem defined over a feasible set $X$ of decision variable vectors assumes the existence of several fuzzy goals $G_{k}, k=1, \ldots, p$ that are fuzzy subsets of $X$ under a set of fuzzy restrictions $R_{i}, i=1, \ldots, m$ that are also fuzzy subsets of $X$. Bellman and Zadeh [1] described a solution to such problem (i.e. a decision), through a fuzzy subset of $X$, i.e. a set $\left\{\left(x, \mu_{D}(x)\right) \mid x \in X\right\}$, where the membership function $\mu_{D}: X \rightarrow[0,1]$ is defined by aggregating the fuzzy goals and restrictions using the min operator

$$
\mu_{D}(x)=\min \left(\left\{\mu_{G_{k}}(x) \mid k=1, \ldots, p\right\} \cup\left\{\mu_{R_{i}}(x) \mid i=1, \ldots, m\right\}\right) .
$$

Further on, Zimmerman [12] proposed the following mathematical problem

$$
\begin{aligned}
\max & \alpha \\
\text { s.t. } & \mu_{G_{k}}(x) \geq \alpha, \quad k=1, \ldots, p \\
& \mu_{R_{i}}(x) \geq \alpha, \quad i=1, \ldots, m \\
& 0 \leq \alpha \leq 1, \\
& x \in X .
\end{aligned}
$$

to derive the optimal decision, namely the solution with the maximal membership value.

Dubey, Chandra and Mehra [4] approached a fuzzy decision problem introducing a bipolar perspective in aggregating the fuzzy goals and constraints. They proposed several linear models to solve fuzzy MO-LP problems, and in Section 4 we extend two of them to models that solve fuzzy MO-LFP problems still conserving their linearity.

\section{Novel membership functions construction}

Let us consider the general form of a deterministic single objective fractional programming problem

$$
\max \left\{f(x)=\frac{N(x)}{D(x)} \mid x \in X\right\} .
$$


In this section we aim to link a fuzzy goal to its objective function $f$, and propose a new way to construct a pair of membership functions to describe the fractional fuzzy goal over its feasible set $X$.

Dutta, Tiwari and Rao [5] introduced a linguistic variable approach to solve MO-LFP maximization problems. They constructed a pair of membership functions, one for the numerator and one for denominator to describe a fractional fuzzy goal. Their idea was to impose the maximal (minimal) value of the numerator (denominator) as threshold for the membership function. Unfortunately, the ratio of the maximal value of the numerator and the minimal value of the denominator is not always sufficiently close to the maximal value of the fraction over the feasible set. This fact was first discussed in [8]. A new pair of thresholds that represent the values of the numerator (denominator) at the point $x^{*}$ that maximizes the fractional objective function of the feasible set $X$ is also proposed in [8].

Adopting the idea of choosing the thresholds in the manner described in [8], we propose a different shape for the membership functions linked to the numerator and denominator of a fractional objective function.

As far as no thresholds are provided for the fractional objective we propose the following triangular shape for the membership function $\mu_{N}$ of the numerator (and $\mu_{D}$ of the denominator)

$$
\mu_{N}(N(x))=\left\{\begin{aligned}
0, & N(x)<N_{\min }, \\
\frac{N(x)-N_{\min }}{N_{0}-N_{\min },} & N_{\min } \leq f(x) \leq N_{0}, \\
1, & N(x)=N_{0} \\
\frac{N(x)-N_{\max }}{N_{0}-N_{\max }}, & N_{0} \leq f(x) \leq N_{\max }, \\
0, & f(x)>N_{\max },
\end{aligned}\right.
$$

where

$$
N_{\min }=\min _{x \in X} N(x), \quad N_{\max }=\max _{x \in X} N(x)
$$

(and $D_{\min }=\min _{x \in X} D(x), D_{\max }=\max _{x \in X} D(x)$ respectively). Using the solution $x^{*}$ that maximizes $N(x) / D(x)$ we compute $N_{0}=N\left(x^{*}\right)$ and $D_{0}=D\left(x^{*}\right)$ for the numerator and denominator respectively. Both membership functions $\mu_{N}$ and $\mu_{D}$ have the same shape, piece-wise monotone, even thought that the first one has to be maximized, while the second one has to be minimized. The reason is the fact that the values greater than $N_{0}$ divided by the values greater than $D_{0}$ can be found in the neighborhood of the best value of the ratio (i.e. from $N_{0} / D_{0}$ ) as well as the values smaller than $N_{0}$ divided by the values greater than $D_{0}$, or the values smaller than $N_{0}$ divided by the values smaller than $D_{0}$. The only impossible combination is to divide values greater than $N_{0}$ by the values smaller than $D_{0}$ since increasing the numerator and decreasing the denominator an increase of the ratio is obtained.

Note that the membership function $\mu_{N}$ in (2) is not defined explicitly at any point $x$ but implicitly using the $y$-values, i.e. $y=N(x)$. Any graphic representation of $\mu_{N}$ with respect to $y$-values is bi-dimensional, while it is $(n+1)$-dimensional with respect to $x$-values, $x$ being an $n$-dimensional vector.

Figure 1 shows the membership functions of numerators and denominators of a fractional objective produced by the novel method and two other methods found in the literature ([5], [8]). The representations are made with respect to $y$-values, thus involving only minimal, maximal, and desired values of the functions. No conclusion can be derived for the membership function of their ratio since no information about the dependence of numerator and denominator through $x$ is provided. On the other side, composing the membership functions with the functions themselves yields representations with respect to the solution space, i.e. over the feasible set. An example of such representations can be seen in Figure 2 that is part of an illustrative example in Section 5.1.

We now turn to the case of a given threshold $p$ smaller than $N_{0} / D_{0}$, and a given tolerance limit $t<p$ both provided for the fractional fuzzy goal. This pair (threshold, tolerance limit) determines four optimization problems (3)

$$
\begin{array}{ll}
N_{1}=\min \{N(x) \mid x \in X, N(x)=t D(x)\}, & N_{4}=\max \{N(x) \mid x \in X, N(x)=t D(x)\} \\
N_{2}=\min \{N(x) \mid x \in X, N(x)=p D(x)\}, & N_{3}=\max \{N(x) \mid x \in X, N(x)=p D(x)\}
\end{array}
$$



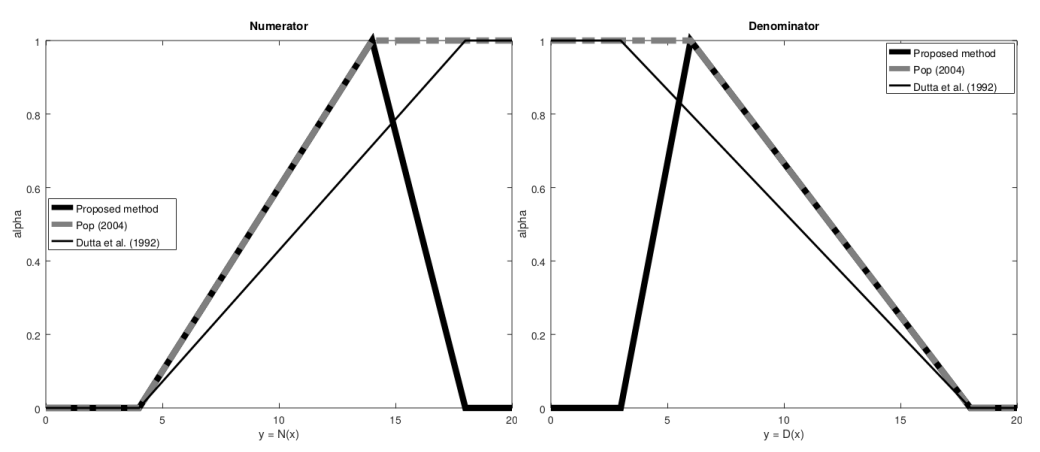

Figure 1: Graphical representation of the membership functions of numerators (to the left) and denominators (to the right) with respect to their $y$-values. The methods from the literature proposed increasing functions for numerators that are maximized and decreasing functions for denominators that are minimized. Our method provides piece-wise monotone functions always containing both increasing and decreasing branches.

that yield the key values for the parameters $N_{1}, N_{2}, N_{3}$ and $N_{4}$ needed in the definition of the trapezoidal shaped membership function of the numerator

$$
\mu_{N}(N(x))=\left\{\begin{aligned}
0, & N(x)<N_{1}, \\
\frac{N(x)-N_{1}}{N_{2}-N_{1}}, & N_{1} \leq f(x)<N_{2}, \\
1, & N(x) \in\left[N_{2}, N_{3}\right] \\
\frac{N(x)-N_{4}}{N_{3}-N_{4}}, & N_{3}<f(x) \leq N_{4}, \\
0, & f(x)>N_{4} .
\end{aligned}\right.
$$

Note that all optimization problems solved in (3) are linear when both numerator $N(x)$ and denominator $D(x)$ are linear. Assuming that the threshold and the tolerance limit for the fractional objective have values in accordance with its range, all values $N_{1}, N_{2}, N_{3}$ and $N_{4}$ are correct defined. Similar computation is needed to determine the parameters $D_{1}, D_{2}, D_{3}$ and $D_{4}$ used in defining the membership function of the denominator. The needed optimization problems are given in (4)

$$
\begin{array}{ll}
D_{1}=\min \{D(x) \mid x \in X, N(x)=t D(x)\}, & D_{4}=\max \{D(x) \mid x \in X, N(x)=t D(x)\} \\
D_{2}=\min \{D(x) \mid x \in X, N(x)=p D(x)\}, & D_{3}=\max \{D(x) \mid x \in X, N(x)=p D(x)\}
\end{array}
$$

The main advantages of the novel pair of membership functions $\left(\mu_{N}, \mu_{D}\right)$ are as follows: they are piece-wise linear, their maximal values are reached at the point at which the maximal value of the ratio is obtained, and their aggregation using min operator is in accordance to the aggregation used in solving classic fuzzy decision problems.

\section{Novel crisp-linear-and models to fuzzy MO-LFP}

Dubey, Chandra and Mehra [4] introduced a crisp-and linear model (CANDLP) to solve fuzzy MO-LP problems. Both the fuzzy goals and restrictions were involved in the same way in the model through their linear membership functions, but they were distinctly treated through distinct fuzzy levels.

We are interested to obtain a linear-and model to solve F-MO-LFP problems. Using fractional objective functions in CANDLP a non-linear model is obtained. To avoid the non-linearity in the extended model, for each objective function

$$
f_{k}(x)=N_{k}(x) / D_{k}(x)=\left(c_{k}^{T} x+c_{k}^{0}\right) /\left(d_{k}^{T} x+d_{k}^{0}\right),
$$

$k=1 \ldots, p$, we introduce the following pair of inequalities

$$
\alpha+\alpha_{k} \leq \mu_{N_{k}}\left(c_{k}^{T} x+c_{k}^{0}\right), \quad \alpha+\alpha_{k} \leq \mu_{D_{k}}\left(d_{k}^{T} x+d_{k}^{0}\right),
$$


where both $\mu_{N_{k}}$ and $\mu_{D_{k}}$ are defined as proposed in Section 3 with respect to the threshold and tolerance given for the fuzzy goal related to the fractional objective function $f_{k}$. In fact, inequalities (5) replace the inequality $\alpha+\alpha_{k} \leq \mu_{G_{k}}\left(c_{k}^{T} x\right)$ that corresponds in CANDLP to the objective $c_{k}^{T} x$.

We may now propose our first extended crisp-linear-and model

$$
\begin{aligned}
& \max \alpha+\frac{1-\gamma}{p+m}\left(\sum_{k=1}^{p} \alpha_{k}+\sum_{i=1}^{m} \alpha_{p+i}\right) \\
& \text { subject to } \alpha+\alpha_{k} \leq \mu_{N_{k}}\left(c_{k}^{T} x+c_{k}^{0}\right), \quad k=1, \ldots, p \text {, } \\
& \alpha+\alpha_{k} \leq \mu_{D_{k}}\left(d_{k}^{T} x+d_{k}^{0}\right), \quad k=1, \ldots, p, \\
& \alpha+\alpha_{k} \leq 1, \quad k=1, \ldots, p, \\
& \alpha+\alpha_{p+i} \leq \mu_{R_{i}}\left(a_{i} x\right), \quad i=1, \ldots, m, \\
& \alpha+\alpha_{p+i} \leq 1, \quad i=1, \ldots, m, \\
& \alpha, \alpha_{k}, \alpha_{p+i} \geq 0, \quad k=1, \ldots, p, i=1, \ldots, m,
\end{aligned}
$$

to solve fuzzy MO-LFP problems. In this model $\gamma$ is a parameter with values in the interval $[0,1]$ used for balancing the weights of the $\alpha$-levels in the objective function. The number of fuzzy constraints in the original problem is denoted by $m$, while all crisp constraints are grouped in the set $X$.

Aiming to make distinction between the fuzzy goals that represent a positive information, and fuzzy restrictions that represent a negative information, Dubey, Chandra and Mehra [4] used distinct aggregation operators for goals on one side and constraints on the other side, thus obtaining their coherence aggregated and linear program (COAANDLP) to solve F-MO-LP problems. This model loses its linearity when fractional objective functions are involved.

Making use of the same pair of membership functions related to a fractional fuzzy goal we extend the applicability of their idea, and introduce the coherence aggregated linear-and model (7) to solving F-MO-LFP problems.

$$
\begin{array}{rlr}
\max & \delta & \\
\text { subject to } & \alpha+\alpha_{k} \leq \mu_{N_{k}}\left(c_{k}^{T} x+c_{k}^{0}\right), & k=1, \ldots, p, \\
& \alpha+\alpha_{k} \leq \mu_{D_{k}}\left(d_{k}^{T} x+d_{k}^{0}\right), & k=1, \ldots, p, \\
& \alpha+\alpha_{k} \leq 1, & k=1, \ldots, p, \\
& \delta \leq \mu_{R_{i}}\left(a_{i} x\right), & \\
& \delta \leq \alpha+\frac{1-\gamma}{p+m} \sum_{k=1}^{p} \alpha_{k}, & \\
& \delta \leq 1, & \\
& \alpha, \alpha_{k}, \delta \geq 0, & k=1, \ldots, p, \\
& x \in X . &
\end{array}
$$

Note the presence of only one acceptability level $\delta$ for all fuzzy constraints, and one special constraint that keeps the acceptability level of the flexible constraints $(\delta)$ under the desirability levels of the goals. This special constraint makes Model (7) to follow the consistency condition [4] which claims that no solution can be unacceptable and desired at the same time.

\section{$5 \quad$ Numerical exemplifications}

\subsection{Illustration of the novel membership function construction}

We first illustrate our novel methodology to construct the pair of membership functions for a linear fractional objective function that has to be maximized over a feasible set. We use the optimization problem

$$
\max \left\{\frac{x_{1}+3 x_{2}+1}{x_{1}+5 x_{2}+5} \mid x_{1}+x_{2} \leq 10,0 \leq x_{1} \leq 8,0 \leq x_{2} \leq 7\right\}
$$

that is part of the MO-LFP problem addressed in [9], where an approximation of the Pareto set was generated. 
Table 1: Extreme points and relevant values for the numerator, denominator and ratio involved in the optimization Problem (8)

\begin{tabular}{|l|c|c|c|c|c||c|l|c|}
\hline Extreme points & $(0,0)$ & $(8,0)$ & $(8,2)$ & $(3,7)$ & $(0,7)$ & $\min$ & desired values & $\max$ \\
\hline Numerator & 1 & 9 & 15 & 25 & 22 & 1 & $N_{0}=N(8,0)=9$ & 25 \\
\hline Denominator & 5 & 13 & 23 & 43 & 40 & 5 & $D_{0}=D(8,0)=13$ & 43 \\
\hline Ratio & 0.2 & 0.692 & 0.652 & 0.581 & 0.55 & 0.2 & $f(8,0)=0.692$ & 0.692 \\
\hline
\end{tabular}
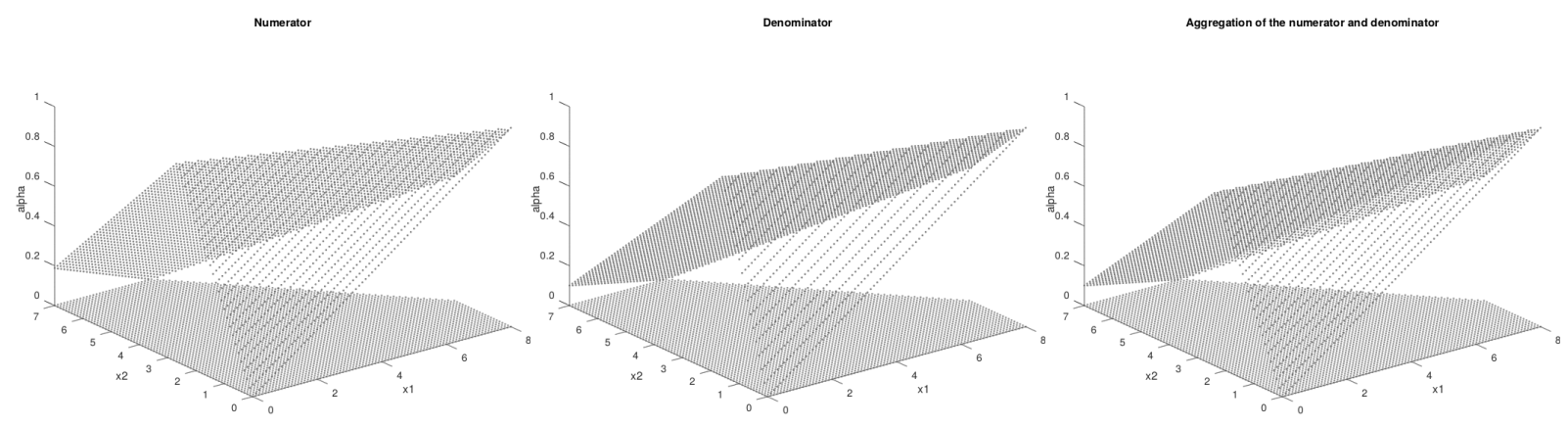

Figure 2: Graphic representations of the numerator, denominator and ratio describing the objective function of Problem (8)

The information about the extreme points of the feasible set and the relevant values used to define the membership functions are provided in Table 1. We use (2) to construct the membership functions since no specific information on the fuzzy goals is given.

The $y$-values used to define $\mu_{N}$ are $(1,9,25)$ while the $y$-values used to define $\mu_{D}$ are $(5,13,43)$, all of them obtained from Table 1, columns (min, desired values, max) respectively.

Figure 2 shows the membership functions of the numerator, denominator and their aggregation over the feasible set. Note that the point with maximal altitude correspond to the same pair $\left(x_{1}, x_{2}\right)$, namely $(8,0)$ in all three graphics of Figure 2 , since they correspond to $N_{0}, D_{0}$ and $N_{0} / D_{0}$ respectively.

\subsection{Illustration of the solution approach}

We next recall the example presented in [3], and adapt it by combining their first and third linear objectives in one fractional objective, and keeping their second objective unchanged, linear. We also keep their constraints unchanged, namely first two constraints are fuzzy with given thresholds and tolerances, while next three constraints are deterministic. The description of the adapted problem is as follows.

A company produces three products $A, B, C$ using three types of raw material $X, Y, Z$. The amount of raw material (in $\mathrm{kg}$ ) required to produce one unit of the products $A, B, C$ is given by the vectors $(2,3,4),(8,1,4)$, and $(4,0,2)$ respectively. There are also some constraints imposed on the quantities of materials produced: it is inappropriate to ask for more than $50 \mathrm{~kg}$ of $X$, and it is acceptable $40 \mathrm{~kg}$ or less; it is not allowed a demand of $Y$ above $55 \mathrm{~kg}$, but it is recommended to use 50 $\mathrm{kg}$ only. These two constraints for $X$ and $Y$ are fuzzy - described by thresholds and tolerances, while the next constraint on $Z$ is hard: the use of material $Z$ above $50 \mathrm{~kg}$ is unacceptable. The next two constraints on producing at least 3 units of $A$, and at least 5 units of $C$ are also hard. We state two goals: to maximize the rate of profit per production costs; and minimize the production of a harmful pollutant. The profit generated by selling one unit of products $A, B$ and $C$ is given by $p=(5,10,12)$; and the costs of production of $A, B$ and $C$ are given by $c=(1,3,4)$. The amount of harmful pollutant per unit production of $A, B$ and $C$ is given by the vector $h=(1,2,1)$. Some a priori fuzzy goals are also stated in [4]: a profit of 150 thousand is desired, but up to 130 thousand is tolerable; the production costs should not be more than 80 thousands, but less than 70 thousand is aimed; it is desired to reduce the production of harmful pollutant to at least $30 \mathrm{~kg}$, but it should not exceed 35 $\mathrm{kg}$. Finally, the problem is to find the optimal production quantities of products $A, B$, and $C$. The 

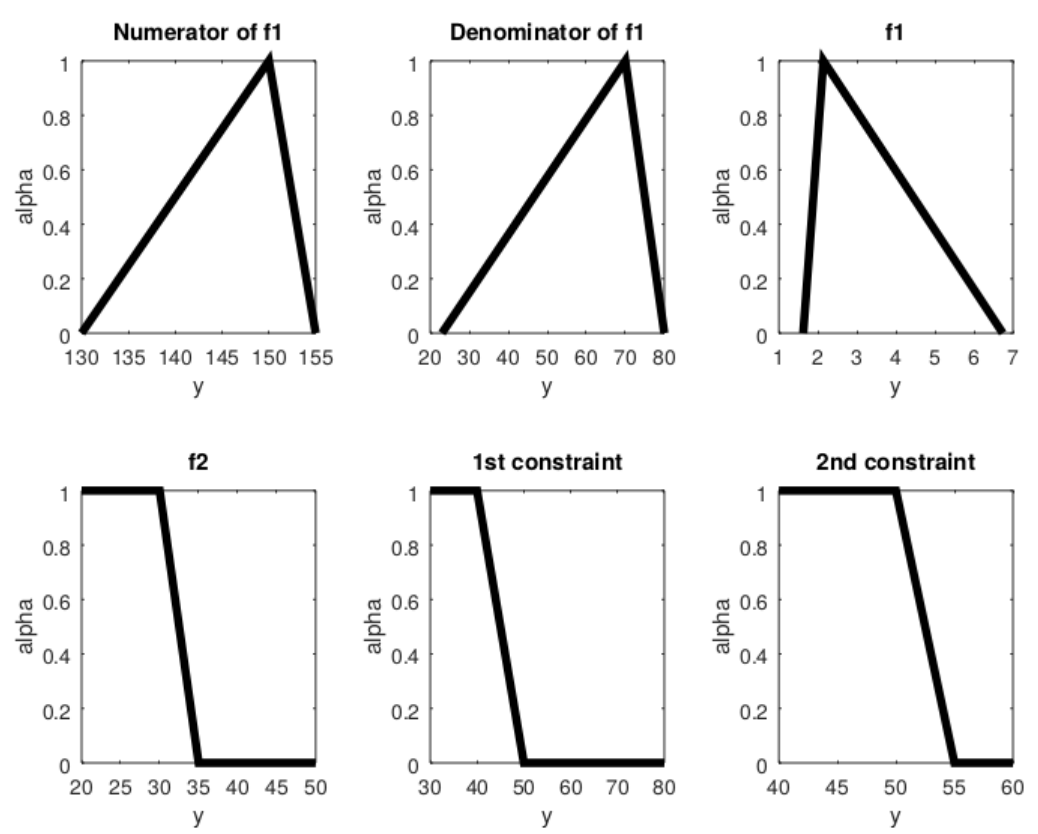

Figure 3: The membership functions of the fuzzy goals and fuzzy constraints involved in Problem (9) with respect to $y$-values

mathematical model of this fuzzy bi-objective optimization problem is

$$
\begin{array}{ll}
\widetilde{\max } & \frac{5 x_{1}+10 x_{2}+12 x_{3}}{x_{1}+3 x_{2}+4 x_{3}} \succeq \frac{150}{70}, \\
\min & x_{1}+2 x_{2}+x_{3} \preceq 30, \\
\text { s.t. } & 2 x_{1}+3 x_{2}+4 x_{3} \preceq 40, \\
& 4 x_{1}+2 x_{3} \preceq 50, \\
& 5 x_{1}+10 x_{2}+12 x_{3} \leq 50, \\
& x_{1} \geq 3, \\
& x_{3} \geq 5, \\
& x_{1}, x_{2}, x_{3} \geq 0 .
\end{array}
$$

The tolerances acceptable for the fuzzy goals and constraints are $g_{1}=20$ for the numerator of $f_{1}$, $g_{2}=10$ for the denominator of $f_{1}, g_{3}=5$ for $f_{2}$, and $r_{1}=10$, and $r_{2}=5$ for the first two constraints respectively. The membership functions of the goals and constraints with respect to $y$-values are shown in Figure 3.

We aim to derive a solution to problem (9) by means of the fuzzy goal programming using the crisp-linear-and models proposed in previous section.

Using Model (6) with $\gamma=0.5$ and the given thresholds and tolerances we obtain the following solution $x_{1}^{*}=3, x_{2}^{*}=6.30488, x_{3}^{*}=5$ with the following levels of satisfactions:

$$
\begin{array}{ll}
\mu_{f_{1}}\left(x^{*}\right)=\alpha^{*}+\alpha_{1}^{*}=0.40244, & \mu_{f_{2}}\left(x^{*}\right)=\alpha^{*}+\alpha_{2}^{*}=1 \\
\mu_{c_{1}}\left(x^{*}\right)=\alpha^{*}+\alpha_{3}^{*}=0.50854, & \mu_{c_{2}}\left(x^{*}\right)=\alpha^{*}+\alpha_{4}^{*}=0.93903 .
\end{array}
$$

Table 2 reports the results obtained for two distinct pairs of thresholds and tolerances for the first objective function, and also those obtained in the absence of any threshold. This numerical results illustrate the dependence of the obtained solution on the desired values and imposed tolerances.

Table 3 reports the results obtained for different pairs of thresholds and tolerances and using Model (7) to solving Problem (9). Note that the third reported solution in both tables is the same. That solution corresponds to the membership functions defined without any specification for the acceptability level. 
Table 2: Optimal solutions obtained by applying Model (6) to solving Problem (9)

\begin{tabular}{|c|c|c|c|c|c|c|}
\hline$x_{1}^{*}$ & $x_{2}^{*}$ & $x_{3}^{*}$ & $\alpha^{*}+\alpha_{1}^{*}$ & $\alpha^{*}+\alpha_{2}^{*}$ & $\alpha^{*}+\alpha_{3}^{*}$ & $\alpha^{*}+\alpha_{4}^{*}$ \\
\hline \hline 3 & 5.88610 & 5.02847 & 0.37813 & 1 & 0.62278 & 1 \\
\hline 3 & 6.30488 & 5 & 0.40244 & 1 & 0.50854 & 0.93903 \\
\hline 3.75 & 0 & 5 & 1 & 1 & 1 & 1 \\
\hline
\end{tabular}

Table 3: Optimal solutions obtained by applying Model (7) to solving Problem (9)

\begin{tabular}{|c|c|c|c|c|c|}
\hline$x_{1}^{*}$ & $x_{2}^{*}$ & $x_{3}^{*}$ & $\alpha^{*}+\alpha_{1}^{*}$ & $\alpha^{*}+\alpha_{2}^{*}$ & $\delta^{*}$ \\
\hline \hline 3 & 4.59839 & 6.07145 & 0.38470 & 1 & 0.42316 \\
\hline 3 & 6.49593 & 5 & 0.41463 & 1 & 0.45122 \\
\hline 3.75 & 0 & 5 & 1 & 1 & 1 \\
\hline
\end{tabular}

\section{Conclusions and final remarks}

In this work we introduced a novel way to construct two piece-wise linear membership functions needed to describe a fuzzy goal linked to a linear fractional objective. The main advantages of the proposed pair of membership functions are their effectiveness in describing the fractional objective still being piece-wise linear; and the fact that their aggregation using min operator is in accordance to the aggregation used in solving classic fuzzy decision problems.

Our second goal was to introduce two crisp linear models to solve fuzzy multiple objective linear fractional programming problems. With the help of the fuzzy-and operator we aggregated all fuzzy goals and constraints in both the classic and bipolar framework; and formulated the crisp linear models yielding a desired solution to the initial fuzzy programming problem. The main advantages of using the new models lies in the fact that they are linear, thus not cumbersome; and can generate distinct solutions to the multiple objective problem by varying the thresholds and tolerance limits imposed on the fuzzy goals, thus accurate.

The membership functions we proposed may have an important role in handling the fractional objectives, thus we aim to study how to involve them in a wider class of solution approaches to fractional optimization problems as an alternative to classic linearization methods. We also aim to develop alternative linear solving models to the same multiple objective linear fractional programming problems involving the fuzzy-or operator or other ordered weights aggregation (OWA) operators. Both these directions are of interest in future researches.

\section{Acknowledgments}

This research was partly supported by the Ministry of Education and Science, Republic of Serbia.

\section{References}

[1] Bellman, R.E.; Zadeh, L.A. (1970). Decision-making in a fuzzy environment. Management Science, 17(4), B-141-B-164.

[2] Chang, C.-T. (2017). Fuzzy linearization strategy for multiple objective linear fractional programming with binary utility functions. Computers $\&$ Industrial Engineering, 112, 437 - 446.

[3] Dubey, D.; Chandra, S.; Mehra, A. (2015). Computing a Pareto-optimal solution for multiobjective flexible linear programming in a bipolar framework. International Journal of General Systems, 44(4), 457-470.

[4] Dubey, D.; Mehra, A. (2014). A bipolar approach in fuzzy multi-objective linear programming. Fuzzy Sets and Systems, 246, 127 - 141. Theme: Preference Modeling and Optimization. 
[5] Dutta, D.; Tiwari, R.N.; Rao, J.R.(1992). Multiple objective linear fractional programming - A fuzzy set theoretic approach. Fuzzy Sets and Systems, 52(1), $39-45$.

[6] Lachhwani, K. (2015). Modified FGP approach for multi-level multi objective linear fractional programming problems. Applied Mathematics and Computation, 266, 1038 - 1049.

[7] Li, D.; Leung, Y.; Wu, W. (2019). Multiobjective interval linear programming in admissible-order vector space. Information Sciences, 486, 1 - 19.

[8] Pop, B. (2004). Fuzzy approach for multiple criteria fractional optimisation - comments and quadratic constraint generalization. Fuzzy Systems and A.I., 10(1-2), $57-65$.

[9] Stanojević, B.; Stanojević, M. (2013). On the efficiency test in multi-objective linear fractional programming problems by Lotfi et al. 2010. Applied Mathematical Modelling, 37(10), 7086 - 7093.

[10] Stanojević, B.; Stanojević, M. (2014). Comment on 'Fuzzy mathematical programming for multi objective linear fractional programming problem'. Fuzzy Sets and Systems, 246, 156 - 159.

[11] Toksari, M.D.; Bilim, Y. (2015). Interactive fuzzy goal programming based on jacobian matrix to solve decentralized bi-level multi-objective fractional programming problems. International Journal of Fuzzy Systems, 17(4), 499-508.

[12] Zimmermann, H.-J. (1978). Fuzzy programming and linear programming with several objective functions. Fuzzy Sets and Systems, 1(1), $45-55$.

[13] Zimmermann, H.-J. (1985). Applications of fuzzy set theory to mathematical programming. Information Sciences, 36(1), 29 - 58.

\section{(c) (i) (9)}

Copyright (C)2020 by the authors. Licensee Agora University, Oradea, Romania.

This is an open access article distributed under the terms and conditions of the Creative Commons Attribution-NonCommercial 4.0 International License.

Journal's webpage: http://univagora.ro/jour/index.php/ijccc/

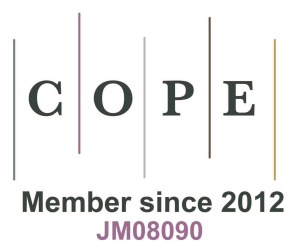

This journal is a member of, and subscribes to the principles of,

the Committee on Publication Ethics (COPE).

https://publicationethics.org/members/international-journal-computers-communications-and-control

Cite this paper as:

Stanojević, B.; Dzitac, S.; Dzitac, I (2020). Crisp-linear-and Models in Fuzzy Multiple Objective Linear Fractional Programming, International Journal of Computers Communications \& Control, 15(1), 1005, 2020. https://doi.org/10.15837/ijccc.2020.1.3812 